\title{
ANÁLISE DA EFICIÊNCIA DE LOCALIZADORES DE ARMADURA ELETROMAGNÉTICOS: UM ESTUDO COMPARATIVO
}

\section{Analysis of the efficiency of electromagnetic reinforcement locators: a comparative study}

\author{
Diego Jesus de Souza ${ }^{1}$, Marcelo Henrique Farias de Medeiros ${ }^{2}$
}

Recebido em 08 de março de 2017; recebido para revisão em 15 de maio de 2017;

aceito em 11 de agosto de 2017; disponível on-line em 19 de setembro de 2017.

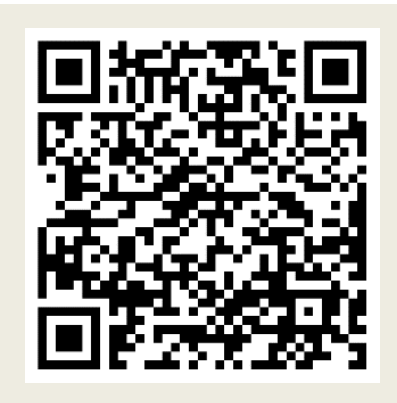

PALAVRAS CHAVE:

Ensaios não destrutivos; Pacometria; Inspeção.

\section{KEYWORDS:}

Non-destructive tests; Pacometry; Inspection.

\footnotetext{
* Contato com o autor:

${ }^{1}$ e-mail: diegojesusdesouza@hotmail.com ( D. J. de Souza)

Mestre em Engenharia Civil, Ph.D. student at University of Ottawa, Ontario, Canada.

22e-mail: medeiros.ufpr@gmail.com (M. H. F. Medeiros)

Doutor em Engenharia Civil, Professor Adjunto, Universidade Federal do Paraná.
}

\begin{abstract}
RESUMO: Na inexistência dos projetos executivos é possível determinar a armadura da estrutura e o seu respectivo cobrimento a partir de aparelhos denominados pacômetros. O presente trabalho tem como objetivo analisar as leituras de equipamentos de dois modelos, o Profometer 5+ e o Wallscanner D-Tect 150, utilizados na detecção das armaduras e cobrimento de um dos pilares do viaduto em Curitiba. A metodologia

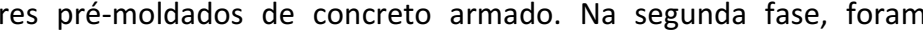
exutadas leituras em um pilar de um edifício comercial e finalmente, na terceira etapa ram realizadas medições em um pilar com armação desconhecida localizado no 位 das barras. Não foram detectadas armaduras localizadas em camadas mais profundas. s dois aparelhos permitiram a estimativa do cobrimento das barras detectadas, (1) a leitura foi bastante afetada em zonas de alta concentração de armadura. Os ce facilidade de uso dos equipamentos, permitiram concluir que é recomendável a utilização de pacômetros em inspeções preliminares de pilares de estruturas de concreto armado.
\end{abstract} ABSTRACT: In the absence of the executive projects it is possible to determine the
armature of the structure and its respective covering from devices called pacemakers.
The present work has the objective of analyzing the equipment readings of two models,
Profometer 5+ and Wallscanner D-Tect 150, used in the detection of the reinforcement
and covering of one of the columns of the bridge in Curitiba. The methodology developed
was divided into three stages, the first one, for equipment calibration, performed on
precast concrete columns. In the second phase, readings were performed on a column of
a commercial building and finally, in the third stage measurements were made on a
column with unknown frame located on the viaduct. The readings showed the efficiency
of the equipment in the location of the reinforcement, but presented little precision in the
determination of the bar diameters. No reinforcement was found located in deeper
layers. The two devices allowed the estimation of the covering of the detected bars,
however, the reading was very affected in zones of high concentration of reinforcement.
The results obtained, combined with the lightness and ease of use of the equipment,
allowed us to conclude that the use of pacometers in preliminary inspections of pillars of
reinforced concrete structures is recommended. 


\section{INTRODUÇÃO}

A execução de ensaios não destrutivos em estruturas de concreto armado, realizados de uma maneira eficiente, permite coletar dados que caracterizem melhor a edificação, complementando as inspeções visuais e disponibilizando mais informações para avaliação do elemento estrutural.

Segundo Sahuinco (2011), os ensaios não destrutivos e semidestrutivos servem para obter informações e consequentemente tomar decisões, tanto em situações em que houver ou não deteriorações aparentes na estrutura, e com um baixo custo de execução.

De acordo com o boletim do $\mathrm{ACl} 228.2 \mathrm{R}-$ 98, os métodos de ensaio não destrutivos estão cada vez mais utilizados em investigações de estruturas de concreto, devido aos avanços tecnológicos dos equipamentos de coleta e análise de dados, pelas vantagens econômicas na avaliação de grandes volumes de materiais em comparação com métodos perfurantes e pela capacidade de realizar avaliações rápidas e abrangentes em construções existentes, fornecendo informações sobre as propriedades no local de moldagem do concreto endurecido.

A pacometria tem como princípio de funcionamento a leitura de correntes eletromagnética que permitem a localização da posição da armadura de aço, assim como uma estimativa do seu diâmetro e a espessura de cobrimento do concreto da estrutura inspecionada. Segundo Santos (2008), o ensaio é baseado na leitura da interação entre uma frequência baixa de um campo eletromagnético criado pelo equipamento e a armação detectada. A partir dos dados recolhidos, intensidade e frequência, é possível estimar o diâmetro e o cobrimento das armaduras. No Brasil ainda não há uma norma técnica que regulamente o uso do pacômetro, de modo que os fabricantes dos equipamentos se baseiam em normas estrangeiras, como a British Standard BS1881: Part 204: Recommendations on the use of electromagnetic covermeters.

Segundo a BS1881: Part 204, pacômetros eletromagnéticos podem ser utilizados para garantir a correta localização e cobrimento de armaduras existentes, cujas características não estão disponíveis, evitando a execução de ensaios invasivos na estrutura inspecionada. A norma ainda descreve que as medições podem ser afetadas por fatores tais como, a presença de diferentes tipos de aço na estrutura, cruzamentos e densidades elevadas de barras na área levantada, presença de arames, pregos ou outros elementos metálicos próximos à superfície do elemento.

O presente trabalho tem como objetivo analisar a eficiência leituras de dois tipos de detectores magnéticos, o Profometer $5+$ e o Wallscanner D-Tect 150, em três diferentes situações: (1) em elementos cujo cobrimento, espaçamento e bitola das barras são conhecidos por medições diretas; (2) elementos de concreto armado produzidos com elevada precisão construtiva, porém às informações das do elemento são conhecidas apenas por projetos; e (3) sem conhecimento de qualquer informação da composição da obra.

\section{PROCEDIMENTO EXPERIMENTAL}

\subsection{EQUIPAMENTOS UTILIZADOS}

O ensaio não destrutivo para detecção de armaduras e cobrimentos de estruturas de concreto armado foi desenvolvido utilizando-se dois instrumentos: Wallscanner D-Tect 150 (WDT); e o equipamento Profometer $5+(\mathrm{P} 5+)$ que permitem também a leitura dos diâmetros das barras encontradas.

O equipamento (WTD) permite a leitura de tubos, incluindo encanamento hidráulico dutos de fiação, assim como barras metálicas de aço, cobre ou alumínio em superfícies de concreto. Segundo o fabricante, caso existam vários objetos sobrepostos dentro da área examinada, o equipamento fará a leitura do que se encontra mais próximo da superfície, porém, a apresentação das características encontradas pode divergir das reais, principalmente, em elementos muito finos ou espessos (BOSCH, 2009).

A localização de armaduras utilizando o WDT é feita quando o equipamento passa pelo 
percurso de medição, repetindo o processo até a detecção da linha central da barra na tela do instrumento, marcando em sua face superior o eixo do objeto encontrado.

O método de medição do equipamento P5+ é baseado no princípio da corrente de Focault com indução por impulso, permitindo que a unidade básica localize as armaduras da estrutura, determine os seus diâmetros e o cobrimento do concreto. A sonda do instrumento reage mais sensivelmente às barras paralelas ao seu eixo longitudinal com movimentos na direção ortogonal (PROCEQ SA, 2012).

Para a determinação precisa do diâmetro da barra, é importante não haver influências que possam alterar o resultado, sendo interessante selecionar um local na estrutura onde haja espaçamento suficiente entre as armaduras (PROCEQ SA, 2012).

\subsection{METODOLOGIA}

A metodologia desenvolvida foi dividida em três etapas, a primeira, para calibração dos equipamentos, realizada na sede da empresa Tecnolajes, em Quatro Barras/PR, na superfície de pilares pré-moldados de concreto armado, executados a partir de projetos conhecidos (Figura 1).

Inicialmente, foram coletadas as medidas obtidas com o equipamento WDT, marcando os eixos das armaduras longitudinais e transversais detectadas, e anotando os valores de cobrimentos obtidos no instrumento em 5 (cinco) seções distintas. As marcações foram confrontadas com as barras existentes e amostras nos elementos (Figura 1). Em seguida foram executadas as leituras utilizando o equipamento P5+, anotando os valores obtidos em 5 (cinco) seções dos diâmetros das barras longitudinais e transversais detectadas, novamente comparando as medidas e os valores encontrados com o detalhamento da peça.

A segunda fase do trabalho foi feita em um pilar da estrutura do edifício comercial 7TH AVENUE, ainda em execução. Seguindo exatamente os mesmos critérios da etapa anterior, coletando os dados em 5 (cinco) seções distintas (Figura 2).

A última etapa do trabalho consistiu na realização de leituras em um dos pilares do viaduto da Estação Marechal Floriano, sobre a Linha Verde, localizado em Curitiba. Foram feitas medidas em uma seção do pilar situada a $1600 \mathrm{~mm}$ do nível do solo, numa faixa localizada entre o eixo do elemento e sua extremidade lateral (Figura 3).

Foram anotadas as barras longitudinais e transversais detectadas pelo equipamento WDT, com suas respectivas distâncias e cobrimentos, sendo depois comparadas com as leituras realizadas com o instrumento da P5+. Os diâmetros das armaduras encontradas foram obtidos a partir das medições efetuadas com o P5+.

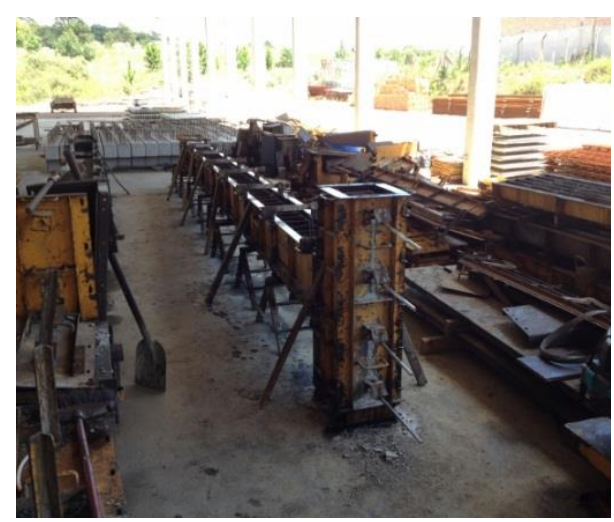

[a]

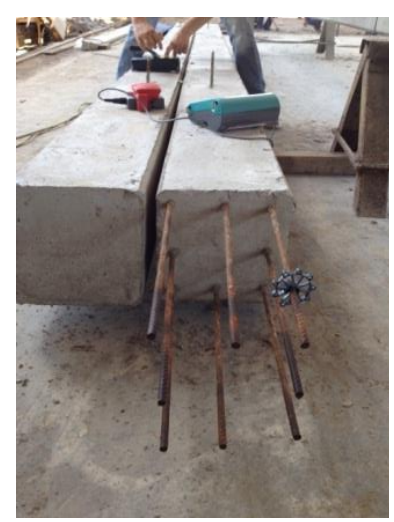

[b]

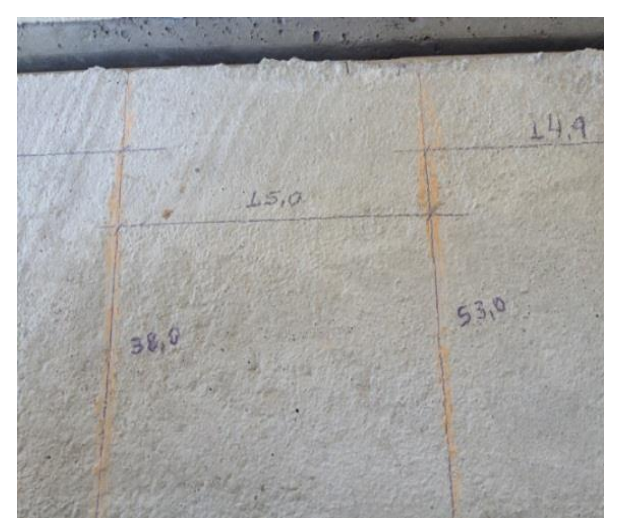

[c]

FIGURA 1: Pilares pré-moldados inspecionados

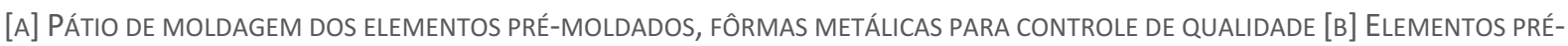
MOLDADOS ONDE FORA REALIZADA A CALIBRAÇÃO DOS EQUIPAMENTOS [C] DEMARCAÇÃO DAS LEITURAS DE ESPAÇAMENTO ENTRE ESTRIBOS. FONTE: Autor. 


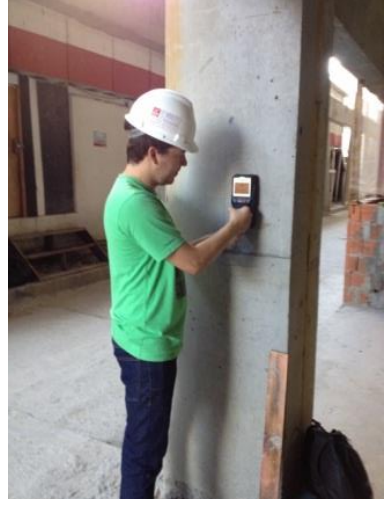

[a]

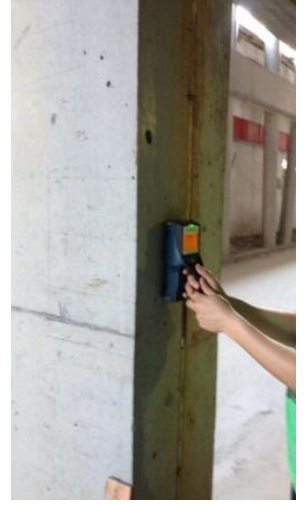

[b]

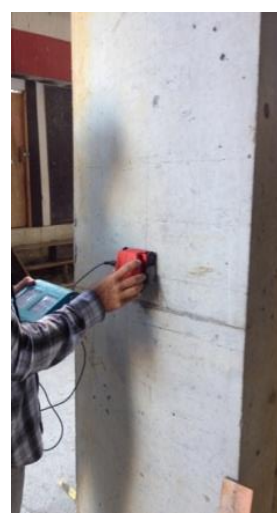

[c]

FIGURA 2: Leituras no pilar P136 com os dois equipamentos utilizados.

[A] REALIZAÇÃO DAS LEITURAS EM BARRAS VERTICAIS DO PILAR P136 UTILIZANDO O EQUIPAMENTO WDT [B] RELEITURAS EM BARRAS VERTICAIS DO PILAR P136 UTILIZANDO O EQUIPAMENTO WDT [C] LEITURAS EM BARRAS VERTICAIS DO PILAR P136 UTILIZANDO O EQUIPAMENTO P5+.

FONTE: Autor.

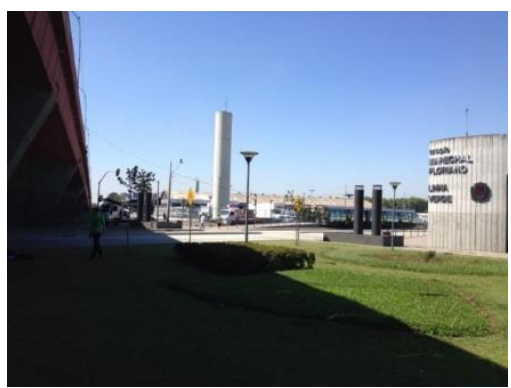

[a]

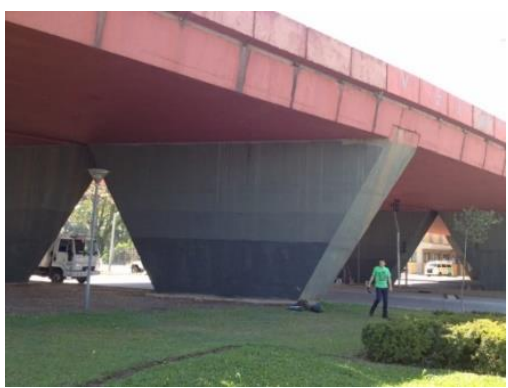

[b]

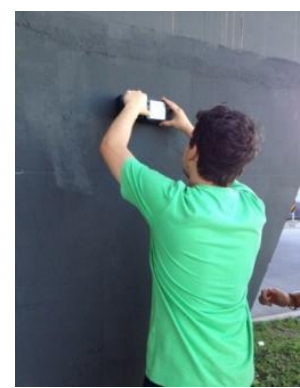

[c]

FIGURA 3: Pilar do viaduto inspecionado

[A] INDICAÇÃO DA LOCALIZAÇÃO DO VIADUTO MARECHAL FLORIANO PEIXOTO INSPECIONADO [B] INDICAÇÃO DOS PILARES PAREDE ONDE FORAM REALIZADOS OS TESTES [C] REALIZAÇÃO DAS LEITURAS EM BARRAS LONGITUDINAIS DO PILAR DO VIADUTO MARECHAL FLORIANO PEIXOTO.

FONTE: Autor.

\section{ANÁLISE DOS RESULTADOS}

\subsection{RESULTADOS OBTIDOS NA CALIBRAÇÃO DOS APARELHOS (MEDIÇÃO EM PILAR PRÉ-MOLDADO).}

Os valores das leituras realizadas em dois pilares pré-moldado, $\mathrm{P} 1$ e $\mathrm{P} 2$, executados com uma seção quadrada de lados $25 \times 25 \mathrm{~cm}$ e com um cobrimento de $30 \mathrm{~mm}$, são apresentados em comparação com os dados coletados diretamente nos elementos, conforme Tabela 1 presentada no ANEXO A.

O aparelho WDT identifica o posicionamento e estima o cobrimento das armaduras reveladas na área inspecionada. As leituras realizadas nos elementos P1 e P2 mostraram as dificuldades do WDT em identificar barras próximas às extremidades das peças examinadas, o instrumento conseguiu detectar apenas a armadura central dos pilares. As informações quanto aos estribos, contudo, se mostraram bastante eficientes, tanto nas posições detectadas, quanto nos espaçamentos obtidos. Os valores obtidos de cobrimento não apresentaram consistência com a realidade (Tabela 1 ).

A análise dos dados coletados mostrou que o aparelho WDT apresentou um erro de apenas $0,27 \%$ para a leitura do espaçamento entre estribos e de aproximadamente $47 \%$ para as medidas de cobrimento das armaduras. As medidas de posicionamento e cobrimento das armaduras realizadas com o aparelho $\mathrm{P} 5+$, mostraram-se mais próximas dos valores reais, não apresentando a limitação na detecção de barras extremas. Porém, observaram-se inconsistências nos valores obtidos para os diâmetros das peças detectadas, mostrando variações nos dados, conforme a velocidade de 
passagem da sonda. Passagens mais lentas produzem leituras mais precisas.

As variações de leitura do aparelho P5+ foram: de 2,7\% para as estimativas de cobrimento e de $0,13 \%$ para a determinação do posicionamento das barras. A obtenção do diâmetro das armaduras gerou um erro de $14,8 \%$. A Figura 4 apresenta a análise comparativa entre médias (teste de Tukey) das leituras aferidas de cobrimento, diâmetro e espaçamento das armaduras.

Logo pode ser observado que, de forma geral, P5+ indica resultados semelhantes aos contidos em projeto para todas as análises feitas, seja de cobrimento, espaçamento ou diâmetro das armaduras. Por outro lado o equipamento WDT não apresenta o mesmo desempenho, ou seja, não apresenta equivalência estatística com os valores de projeto para o pilar um, indicando elevada variabilidade. Porém, para as demais leituras este equipamento mostrou-se satisfatório.

\section{TABELA 1: Comparação entre leituras com os dados de projeto para Pilar P1.}

\begin{tabular}{|c|c|c|c|}
\hline \multirow{2}{*}{ Elemento de leitura } & \multicolumn{3}{|c|}{ Pilar Pré-Moldado P1 } \\
\hline & Dados do elemento & WDT & P5+ \\
\hline \multicolumn{4}{|l|}{ Diâmetro da Armadura do } \\
\hline Estribo $\phi(\mathrm{mm})$ & 5.0 & - & $\phi<7.0$ \\
\hline Cobrimento (mm) & 29,9 & 13,8 & 28,9 \\
\hline Espaçamento (mm) & 150 & 150,4 & 149,8 \\
\hline \multirow{2}{*}{ Elemento de leitura } & \multicolumn{3}{|c|}{ Pilar Pré-Moldado P2 } \\
\hline & Dados de projeto & WDT & P5+ \\
\hline № de barras lidas em uma face & 3 & 1 & 3 \\
\hline$\phi$ Arm. Vertical ESQUERDA (mm) & 10 & - & 10,52 \\
\hline$\phi$ Arm. Vertical CENTRAL (mm) & 10 & - & 11,86 \\
\hline$\phi$ Arm. Vertical DIREITA (mm) & 10 & - & 12,06 \\
\hline Cobrimento (mm) & 30,0 & 18,0 & 30,5 \\
\hline
\end{tabular}

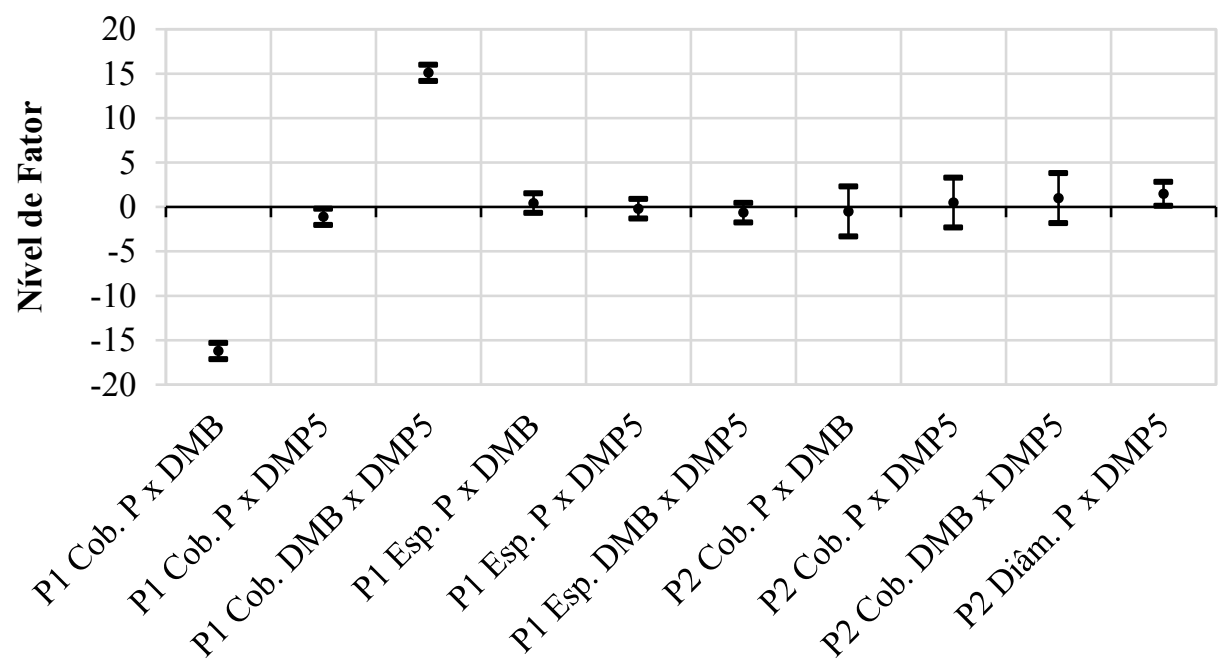

FIGURA 44: Análise comparativa entre médias das leituras aferidas de cobrimento, diâmetro e espaçamento das armaduras para elementos pré-moldados (teste de Tukey). 


\subsection{RESULTADOS OBTIDOS NA MEDIÇÃO DE UM PILAR DE UM EDIFÍCIO}

As leituras realizadas no pilar P136, elemento da estrutura do edifício 7th Avenue, em execução pela construtora Thá Engenharia, são apresentadas na Tabela 2, que compara os dados obtidos com as informações de projeto.

Os resultados dessas leituras apresentaram um aumento nas margens de variações, quando comparadas com os dados obtidos nos elementos pré-moldados. Isso mostra que, apesar do rigoroso controle de qualidade demonstrado na obra, o processo executivo de estruturas moldadas "in loco" provoca uma diminuição na precisão do posicionamento e do cobrimento das armaduras, aumentando as divergências entre as medidas realizadas com os aparelhos de detecção e o projeto da estrutura.

Em relação ao projeto da estrutura, o equipamento WDT apresentou uma variação de $4 \%$ na determinação do posicionamento das armaduras, superior ao erro de $0,27 \%$ obtido na leitura dos pilares prémoldados. Quanto aos valores de cobrimento, foram observadas variações de $16,7 \%$ em relação aos especificados em projeto.

O P5+ apresentou, em relação aos dados de projeto, uma diferença de $0,66 \%$ no posicionamento das barras longitudinais e $0,53 \%$ para as armaduras transversais, superior aos $0,13 \%$ obtidos nas estruturas prémoldadas. $\mathrm{Na}$ determinação das armaduras foi encontrada uma diferença de 10,9\%, frente aos 2,7\% obtidos na estrutura pré-moldada. $\mathrm{Na}$ estimativa do cobrimento foi encontrada uma variação de $29,5 \%$ e na leitura dos diâmetros das barras foi obtida uma diferença média de 5,8\% em relação ao projeto.

A variação de medidas, superior a encontrada nos pilares prémoldados, está relacionada a dois fatores: o erro de medida do aparelho e por possíveis diferenças entre a execução e o especificado em projeto. Com a Figura 5 apresenta a análise comparativa entre médias (teste de Tukey) das leituras aferidas de cobrimento, diâmetro e espaçamento das armaduras. Pode ser visto que de modo geral os equipamentos mostraram variabilidades significativas quando comparados com os valores de projeto. Contudo, tais variações não devem ser apenas acarretadas aos equipamentos testados, afinal, em elementos moldados no local, há sempre a vulnerabilidade às deficiências construtivas, como movimentação de formas e armaduras, falta de espaçadores, etc.

TABELA 2: Comparação entre leituras com os dados de projeto para Pilar P136.

\begin{tabular}{cccc} 
Elemento de leitura & \multicolumn{2}{c}{ Pilar Edifício (Leitura de elementos horizontais) } \\
\cline { 2 - 4 } & Dados de projeto & WDT & P5+ \\
\hline Arm. do Estribo $\phi(\mathbf{m m})$ & 5.0 & - & 07.0 \\
Cobrimento $(\mathbf{m m})$ & 30 & 25 & 46,25 \\
Espaçamento $(\mathbf{m m})$ & 150 & 144 & 147 \\
No barras lidas em uma face & 6 & 6 & 6 \\
$\phi$ Arm. Vertical $\mathbf{1}(\mathbf{m m})$ & 12,5 & - & 11,60 \\
$\phi$ Arm. Vertical $\mathbf{2}(\mathbf{m m})$ & 12,5 & - & 16,7 \\
$\phi$ Arm. Vertical $3(\mathbf{m m})$ & 12,5 & - & 12,75 \\
$\phi$ Arm. Vertical $\mathbf{4}(\mathbf{m m})$ & 12,5 & - & 12,6 \\
$\phi$ Arm. Vertical adotado $(\mathbf{m m})$ & 12,5 & - & 12,5 \\
Cobrimento $(\mathbf{m m})$ & 35 & 31,5 & 39,7 \\
\hline
\end{tabular}




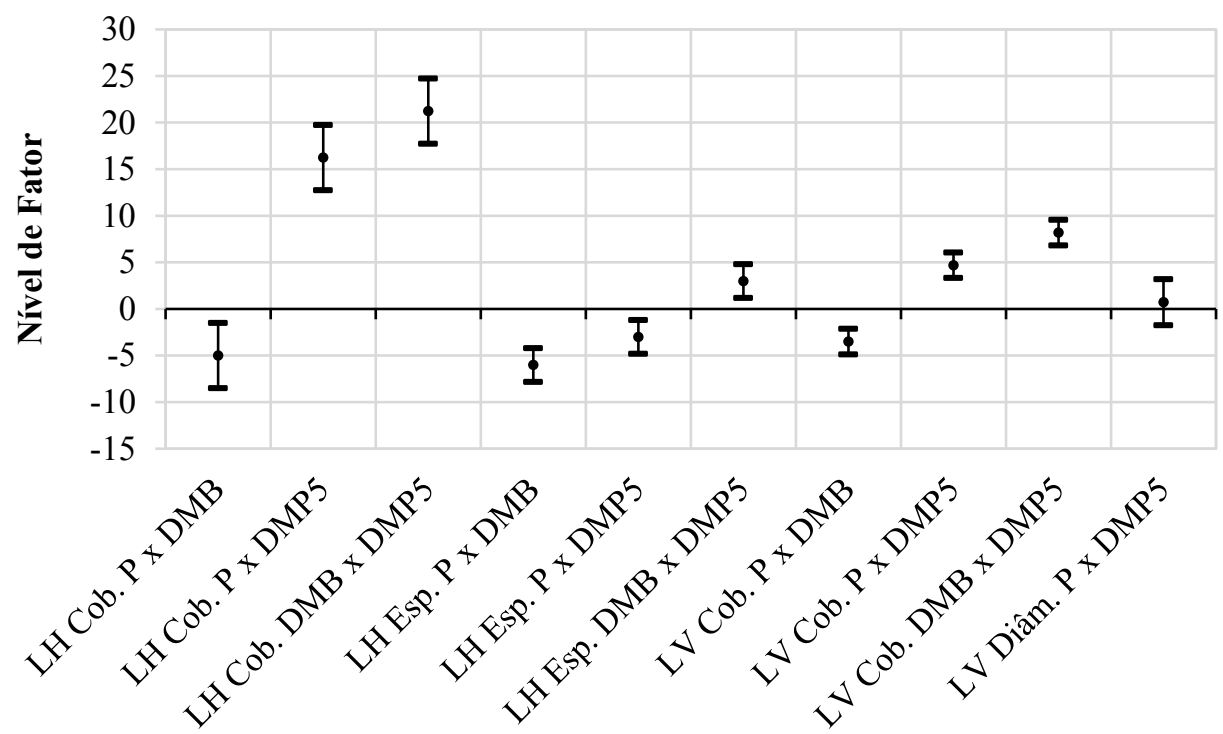

FIGURA 5: Análise comparativa entre médias das leituras aferidas de cobrimento, diâmetro e espaçamento das armaduras para elemento moldado no local (teste de Tukey).

FONTE: Autor.

\subsection{RESULTADOS OBTIDOS NA MEDIÇÃO DO PILAR DE UM VIADUTO}

As leituras das barras longitudinais do pilar do viaduto foram feitas em uma linha de referência, a uma altura de $160 \mathrm{~cm}$ do solo, e as armaduras transversais foram lidas no alinhamento dos eixos 1 , 2 e 3 (Tabela 3). Na área inspecionada, foram localizadas um total de 21 barras de armadura longitudinal vertical e 4 (quatro) na direção transversal. Os valores obtidos são mostrados nas Tabela 4 e Tabela 5.

Durante as inspeções, o Profometer 5+, da PROCEQ, apresentou duas mensagens de erro de leitura, "resultados inválidos" e "cobrimento muito espesso", demonstrando as condições desfavoráveis de alguns pontos. A superfície ondulada e irregular do pilar vistoriado prejudicou as leituras realizadas, dificultando o trajeto das sondas dos equipamentos, impossibilitando a medida em determinados pontos. Trechos com uma provável densidade maior de armaduras também prejudicaram as leituras dos aparelhos. Considerando os valores de calibração obtidos nas medições anteriores, apresenta-se nas Tabela 6 e as estimativas de diâmetros das armaduras detectadas, suas espessuras de cobrimento (C) e os seus espaçamentos (E). 


\begin{tabular}{|c|c|c|c|c|c|c|}
\hline \multirow{3}{*}{$\begin{array}{c}\text { Elementos } \\
\text { Verticais }\end{array}$} & \multicolumn{6}{|c|}{ Pilar Viaduto } \\
\hline & \multicolumn{3}{|c|}{ WDT } & \multicolumn{3}{|c|}{ P5+ } \\
\hline & $\phi(\mathrm{mm})$ & $C(\mathrm{~mm})$ & $E(\mathrm{~mm})$ & $\phi(\mathrm{mm})$ & $\mathrm{C}(\mathrm{mm})$ & $E(\mathrm{~mm})$ \\
\hline Barra 1 & Não Lida & 25 & 0 & 19,1 & 37 & 0 \\
\hline Barra 2 & Não Lida & 19 & 259 & 25 & 40 & 263 \\
\hline Barra 3 & Não Lida & 14 & 118 & 24,4 & 35 & 123 \\
\hline Barra 4 & Não Lida & 8 & 115 & 19,5 & 37 & 127 \\
\hline Barra 5 & Não Lida & 31 & 112 & 27,6 & 47 & 105 \\
\hline Barra 6 & Não Lida & 32 & 129 & 27,1 & 48 & 123 \\
\hline Barra 7 & Não Lida & 31 & 178 & 28,2 & 46 & 177 \\
\hline Barra 8 & Não Lida & 31 & 129 & 25,5 & 45 & 135 \\
\hline Barra 9 & Não Lida & 30 & 137 & 25,3 & 46 & 131 \\
\hline Barra 10 & Não Lida & 28 & 155 & 23,7 & 43 & 159 \\
\hline Barra 11 & Não Lida & 30 & 121 & 24 & 43 & 131 \\
\hline Barra 12 & Não Lida & 27 & 198 & 27 & 41 & 208 \\
\hline Barra 13 & Não Lida & 25 & 116 & 23,3 & 41 & 123 \\
\hline Barra 14 & Não Lida & 31 & 225 & 24,8 & 42 & 210 \\
\hline Barra 15 & Não Lida & 33 & 81 & 24,8 & 42 & 79 \\
\hline Barra 16 & Não Lida & 35 & 119 & 23,9 & 45 & 112 \\
\hline Barra 17 & Não Lida & 55 & 118 & Não Lida & Não Lida & Não Lida \\
\hline Barra 18 & Não Lida & 34 & 176 & 26,1 & 46 & 169 \\
\hline Barra 19 & Não Lida & 34 & 113 & 28,7 & 47 & 110 \\
\hline Barra 20 & Não Lida & 42 & 115 & 33,4 & 54 & 111 \\
\hline Barra 21 & Não Lida & 53 & 110 & Não Lida & Não Lida & Não Lida \\
\hline
\end{tabular}

TABELA 4: Valores encontrados nas leituras horizontais do pilar de viaduto, onde $\phi$ (diâmetro), C (cobrimento) e E (espaçamento entre a barra lida com a anterior).

\begin{tabular}{|c|c|c|c|c|c|c|c|}
\hline \multirow{2}{*}{\multicolumn{2}{|c|}{ Elementos Horizontais }} & \multicolumn{6}{|c|}{ Pilar Viaduto } \\
\hline & & \multicolumn{3}{|c|}{ WDT } & \multicolumn{3}{|c|}{ P5+ } \\
\hline & & $\phi(\mathrm{mm})$ & $\mathrm{C}(\mathrm{mm})$ & $E(\mathrm{~mm})$ & $\phi(\mathrm{mm})$ & $\mathrm{C}(\mathrm{mm})$ & $E(\mathrm{~mm})$ \\
\hline \multirow{4}{*}{ Leitura 1} & Barra 1 & Não Lida & 25 & 0 & 12,4 & 48 & 0 \\
\hline & Barra 2 & Não Lida & 47 & 179 & 9,1 & 54 & 171 \\
\hline & Barra 3 & Não Lida & 29 & 168 & Não Lida & Não Lida & Não Lida \\
\hline & Barra 4 & Não Lida & 20 & 155 & Não Lida & Não Lida & Não Lida \\
\hline \multirow{4}{*}{ Leitura 2} & Barra 1 & Não Lida & Não Lida & Não Lida & 11,4 & 28 & 0 \\
\hline & Barra 2 & Não Lida & Não Lida & Não Lida & Não Lida & Não Lida & Não Lida \\
\hline & Barra 3 & Não Lida & 35 & 0 & Não Lida & Não Lida & Não Lida \\
\hline & Barra 4 & Não Lida & 28 & 144 & Não Lida & Não Lida & Não Lida \\
\hline \multirow{4}{*}{ Leitura 3} & Barra 1 & Não Lida & 27 & 0 & 26,5 & 36 & 0 \\
\hline & Barra 2 & Não Lida & 27 & 162 & 31,2 & 55 & 159 \\
\hline & Barra 3 & Não Lida & 30 & 144 & 34,1 & 57 & 148 \\
\hline & Barra 4 & Não Lida & 30 & 153 & $>41$ & 39 & 152 \\
\hline
\end{tabular}




\begin{tabular}{|c|c|c|c|}
\hline \multirow{3}{*}{$\begin{array}{l}\text { Elementos } \\
\text { Verticais }\end{array}$} & \multicolumn{3}{|c|}{ Pilar Viaduto } \\
\hline & \multicolumn{3}{|c|}{ Valores Adotados } \\
\hline & $\phi(\mathrm{mm})$ & $C(\mathrm{~mm})$ & $E(\mathrm{~mm})$ \\
\hline Barra 1 & 22,2 & 40 & 0 \\
\hline Barra 2 & 22,2 & 40 & 250 \\
\hline Barra 3 & 22,2 & 40 & 120 \\
\hline Barra 4 & 22,2 & 40 & 120 \\
\hline Barra 5 & 25 & 40 & 120 \\
\hline Barra 6 & 25 & 40 & 120 \\
\hline Barra 7 & 25 & 40 & 150 \\
\hline Barra 8 & 25 & 40 & 120 \\
\hline Barra 9 & 25 & 40 & 120 \\
\hline Barra 10 & 25 & 40 & 150 \\
\hline Barra 11 & 25 & 40 & 120 \\
\hline Barra 12 & 25 & 40 & 150 \\
\hline Barra 13 & 22,2 & 40 & 120 \\
\hline Barra 14 & 22,2 & 40 & 200 \\
\hline Barra 15 & 22,2 & 40 & 100 \\
\hline Barra 16 & 22,2 & 40 & 120 \\
\hline Barra 17 & Não Lida & 40 & 120 \\
\hline Barra 18 & 25 & 40 & 150 \\
\hline Barra 19 & 25 & 40 & 120 \\
\hline Barra 20 & 32 & 40 & 120 \\
\hline Barra 21 & Não Lida & 40 & 120 \\
\hline
\end{tabular}

\section{Tabela 6: Valores adotados para as armaduras transversais do pilar.}

\begin{tabular}{lrccc}
\multirow{2}{*}{ Elementos Horizontais } & \multicolumn{3}{c}{ Vilar Viaduto } \\
\cline { 3 - 5 } & & $\phi(\mathbf{m m})$ & $\mathbf{C}(\mathbf{m m})$ & E (mm) \\
\cline { 2 - 5 } & Barra 1 & 10 & 40 & 0 \\
Leitura 1 & Barra 2 & 10 & 40 & 150 \\
& Barra 3 & Não Lida & 40 & 150 \\
& Barra 4 & Não Lida & 40 & 150 \\
& Barra 1 & 10 & Não Lida & Não Lida \\
Leitura 2 & Barra 2 & Não Lida & Não Lida & Não Lida \\
& Barra 3 & Não Lida & 40 & 0 \\
& Barra 4 & Não Lida & 40 & 150 \\
& Barra 1 & 22,2 & 40 & 0 \\
& Barra 2 & 25 & 40 & 150 \\
Leitura 3 & Barra 3 & 32 & 40 & 150 \\
& Barra 4 & 32 & 40 & 150 \\
\hline & & & & FONTE: Autor.
\end{tabular}




\section{RECOMENDAÇÕES FINAIS}

Os resultados obtidos, aliados à leveza e facilidade de uso dos equipamentos, permitiram concluir que é recomendável a utilização de pacômetros em inspeções preliminares de pilares de estruturas de concreto armado.

A detecção do posicionamento e espaçamento das armaduras mostrou-se eficiente nas leituras realizadas pelos dois equipamentos estudados, os dois aparelhos tiveram resultados próximos. O WDT, inicialmente, apresentou dificuldades na determinação de barras localizadas nos extremos dos pilares. A solução adotada foi a utilização de uma placa de madeira, colocada no alinhamento da superfície de leitura, que permitiu a continuidade do movimento da sonda do aparelho até o limite do pilar.

A estimativa do cobrimento apresentou leituras muito variáveis, com maior precisão nas medidas realizadas no aparelho $\mathrm{P} 5+$, com erro máximo de 29,5\%. O WDT apresentou um desvio de $47,0 \%$. Recomenda-se, para melhorar essa avaliação, a execução de uma quantidade de medições maiores, que permita um tratamento estatístico dos dados, obtendo valores mais próximos da realidade.

As medidas relacionadas com o diâmetro das barras também apresentaram variações, relacionadas, principalmente, com a proximidade das armaduras detectadas. Apenas o aparelho P5+ permite essa leitura, obtendo um erro máximo de $33,9 \%$. A execução de leituras em alturas variadas, afastadas do topo e da base dos pilares, regiões com maior densidade de armaduras, também em quantidades adequadas, com um tratamento estatístico que permita resultados mais próximos dos diâmetros existentes.

A BS 1881: Part 204, apesar de permitir correções de leituras baseadas na experiência dos responsáveis pela inspeção, não define um procedimento para isso. Inspeções em condições desfavoráveis, relacionadas com densidades elevadas de armaduras, ondulações e rugosidades na superfície estudada, necessitam de calibrações eficientes, elaboradas em elementos estruturais similares.

A utilização de ensaios não destrutivos, como a leitura de armaduras realizadas com pacômetros, pode se tornar mais eficiente quando associada com técnicas invasivas de inspeção. Dessa forma, a calibração das medidas poderá ser feita considerando as características da estrutura em estudo, permitindo estimativas mais precisas.

\section{REFERÊNCIAS BIBLIOGRÁFICAS}

ABNT - ASSOCIAÇÃO BRASILEIRA DE NORMAS TÉCNICAS. NBR 6118- Projeto de Estruturas de Concreto Procedimento. Rio de Janeiro, 2003.

BS 1881: Part 204. Recommendations on the use of electromagnetic covermeters. 1988.

AMERICAN CONCRETE INSTITUTE. ACI 228.2R-98, Nondestructive Test Methods for Evaluation of Concrete in Structure, 1998.

PROCEQ SA. Instruções operacionais do equipamento Profometer 5+ Modelo Scanlog - Sistema de detecção de barras de reforço. Suiça: 2012.

ROBERT BOSCH GMBH. Instruções operacionais do equipamento WallscannerD-Tectt 150. Alemanha: 2009.

SANTOS, J. M. M. N. Avaliação da integridade estrutural de pontes de betão: 0 caso da Ponte Nossa Senhora da Guia. Dissertação de Mestrado, FEUP, Porto: 2008. 